\title{
Traditional Chinese Medicine Aconiti Radix Cocta Improves Rheumatoid Arthritis via Suppressing COX-1 and COX-2
}

\author{
Shuang Li, ${ }^{1}$ Rui Li, ${ }^{1}$ Ya-Xin Xu, ${ }^{2}$ Jan P. A. Baak, ${ }^{3}$ Ji-Hai Gao, ${ }^{1}$ Jian-Qin Shu, ${ }^{1}$ Li-Jia Jing, \\ Xian-Li Meng, ${ }^{1}$ Chuan-Biao Wen $\mathbb{D}^{\mathbb{D}},{ }^{2}$ Yan-Xiong Gan $\mathbb{D}^{\mathbb{D}},{ }^{2}$ Shi-Chao Zheng $\mathbb{D}{ }^{2}$ \\ and Yong Zeng ${ }^{1}$ \\ ${ }^{1}$ School of Pharmacy, Chengdu University of Traditional Chinese Medicine, Chengdu 611137, China \\ ${ }^{2}$ School of Medical Information Engineering, Chengdu University of Traditional Chinese Medicine, Chengdu 611137, China \\ ${ }^{3}$ Department of Pathology, and Department of Research,, Stavanger University Hospital, Dr. Jan Baak AS, Risavegen 66, \\ Stavanger 4056, Norway
}

Correspondence should be addressed to Yan-Xiong Gan; ganyanxiong@cdutcm.edu.cn, Shi-Chao Zheng; zhengshichao@ cdutcm.edu.cn, and Yong Zeng; zengy1976@163.com

Received 11 January 2021; Revised 17 May 2021; Accepted 15 August 2021; Published 8 September 2021

Academic Editor: Silvia Wein

Copyright $\odot 2021$ Shuang Li et al. This is an open access article distributed under the Creative Commons Attribution License, which permits unrestricted use, distribution, and reproduction in any medium, provided the original work is properly cited.

\begin{abstract}
According to Traditional Chinese Medicine (TCM), Aconiti Radix Cocta (AC) is clinically employed to expel wind, remove dampness, and relieve pain. We evaluated the antirheumatoid arthritis (RA) activities and underlying mechanisms of AC. The chemical constituents of AC were analyzed by high-performance liquid chromatography (HPLC) using three reference compounds (benzoylaconitine, benzoylmesaconine, and benzoylhypacoitine). The anti-RA effects of AC were evaluated in adjuvant-induced arthritis (AIA) rats by hind paw volume and histopathological analysis. The effects of AC on inflammatory cytokines (IL-1 $\beta$ and IL-17A) were determined by enzyme-linked immunosorbent assay. The regulation of cyclooxygenases (COX-1 and/or COX-2) was determined by Western blot and real-time quantitative reverse transcription polymerase chain reaction analyses. AC significantly reduced paw swelling, attenuated the inflammation and bone destruction in joint tissues, and reduced IL-1 $\beta$ and IL-17A in the serum. Moreover, AC downregulated the expression of COX-1 and COX-2 in the synovial tissues. We also identified that AC possesses significant anti-RA activities on AIA, which may be ascribed to the regulation of inflammatory cytokines IL-1 $\beta$ and IL-17, as well as to the inhibition of arachidonic acid signaling pathways. Our findings provide theoretical support for AC as an effective nature-derived therapeutic agent for RA treatment.
\end{abstract}

\section{Introduction}

Rheumatoid arthritis (RA) is a polyarticular (often but always) symmetric disease that involves multiple joints bilaterally, characterized by progressive joint destruction and deformity [1]. Without sufficient treatment, RA can cause accumulating joint damage, dysfunction, and poor physical functioning, leading to inefficient work capacity, low quality of life, and even death in severe cases [2,3]. Worldwide, RA is a frequent disease (prevalence of RA is approximately $0.5 \%$ and the age-standardized prevalence and incidence rates are increasing), thus rendering this autoimmune and inflammatory disease as a major public health challenge [4]. Therefore, early treatment of RA is crucial. At present, two main medical treatments are considered for RA [5]: diseasemodifying antirheumatic drugs (DMARDs) and nonsteroidal anti-inflammatory drugs (NSAIDs). DMARDs, such as methotrexate (MTX), can reduce inflammation, relieve pain, suppress disease activity, and slow cartilage/bone destruction. MTX was the first DMARDs to be approved, and it is currently the most commonly used DMARD for RA patients, as the first-line anchored DMARD to treat RA in clinical settings [6]. However, long-term use of MTX may cause severe systemic complications. In contrast, NSAIDs have been widely used to relieve pain and other inflammatory diseases in clinical settings [7]. However, prolonged use of NSAIDs is linked to the manifestation of several adverse side effects such as gastrointestinal distress, high 
blood pressure, and cardiovascular complications. In addition to their side effects, both DMARDS and NSAIDs are limited by their high cost. Therefore, it is especially urgent to identify and introduce a novel medicinal plant as an alternative to the current treatment regimes, which will be regarded as both safe and effective on RA.

With over thousands of years of history, Traditional Chinese Medicine (TCM) has been proposed as an alternative therapy to effectually alleviate chronic joint inflammation in arthritis, due to its long medicinal history, strong medical value, mild toxic side effects, and affordable prices [8]. Aconiti Radix Cocta (AC) is a dried mother root of Aconitum carmichaelii Debx. that was first recorded in the earliest Chinese medicinal classic work, Shennong's Materia Medica. AC has been used for the treatment of rheumatism and joint pain, due to its beneficial effects of expelling wind and removing dampness, but also in terms of warming menstruation and relieving pain [9]. AC is the principal herb of Wutou decoction, a representative TCM formula for RA treatment with promising clinical efficacy [10]. Wutou decoction could reverse the abnormal suppression of the peroxisome proliferator-activated receptorgamma (PPAR- $\gamma$ ) coactivator pathway in thermogenesis in collagen-induced arthritis rats with wind-cold-dampness stimulus [11].

The main chemical components of AC are benzoylaconitine, benzoylmesaconine, and benzoylhypacoitine, three major alkaloids with a C19-diterpenoid skeleton. The content range of these principal active ingredients in AC is specified in The Pharmacopoeia of the People's Republic of China [12]. All three components have exhibited distinct anti-inflammatory effects on lipopolysaccharide-stimulated macrophages. In particular, benzoylaconitine has shown significant anti-inflammatory activities in in vivo experiments, which can reduce primary and secondary paw swelling in AIA rats, and has also demonstrated a therapeutic effect on collagen-induced arthritis [13, 14]. These results indicate that the $\mathrm{AC}$ components have a prominent anti-inflammatory potential that can also be used effectively in treating arthritis. However, pharmacological studies on the antiarthritic ability of AC on RA are few. Moreover, the mechanism of reducing inflammation is still unclear. Therefore, it is important to elucidate the mechanism of AC on RA.

In our previous study on protein network [15], the antiinflammatory action of AC was found to be related to prostaglandins (PGs) synthesis. PGs are metabolites of arachidonic acid via the cyclooxygenase (COX) pathway. COX is a key enzyme that catalyzes the conversion of arachidonic acid to PGs including COX-1 and COX-2 [16]. COX-1 is a structural enzyme that is essential for maintaining human physiological needs [17], whereas COX-2 is an inducible enzyme that is highly expressed during inflammation [18]. COX-2 can enhance PGE2 synthesis during inflammation, which plays a significant role in the subsequent cell inflammatory responses [19], eventually produce a series of inflammatory mediators, and participate in the body's physiological and pathological processes through a cascade reaction. Research has shown that high levels of COX-2 and
PGs are detected in synovial fibroblasts obtained from RA patients. Results have confirmed that the increase of PGs content is due to the increased expression of COX-2 [20]. Therefore, we hypothesized that the AC mechanism of action may be related to COX inhibition and thus to the inhibition of the conversion of arachidonic acid to PGs.

The classical complete Freund's adjuvant-induced arthritis (AIA) model resembles human RA damage and is characterized by a rapid onset and progression to polyarticular inflammation. It is a model of chronic inflammation, which is $\mathrm{T}$ cell-mediated that shares some features with human RA, including joint swelling, cartilage degradation, loss of joint function, and lymphocyte infiltration of the joints $[21,22]$. These properties render this model as suitable for inducing arthritis. Therefore, an AIA rat model was employed to evaluate the effects of AC and its potential pharmacological mechanism on RA treatment.

\section{Materials and Methods}

2.1. Preparation of Samples. AC was purchased from Sichuan Neautus Traditional Chinese Medicine Co., Ltd. (Sichuan, China) (Number: D1905110). $50 \mathrm{~g} \mathrm{AC}$ was soaked in $500 \mathrm{~mL}$ pure water for $30 \mathrm{~min}$ and boiled for $1 \mathrm{~h}$, gaining the decoction. Then $400 \mathrm{~mL}$ pure water was added and boiled for another $1 \mathrm{~h}$, gaining the second decoction. The filtered decoction was combined and concentrated to $50 \mathrm{~mL}$ to make $1 \mathrm{~g} / \mathrm{mL}$ drug stocks and diluted with pure water to proper concentration for in vivo study.

Benzoylaconitine, benzoylmesaconine, and benzoylhypacoitine reference standards were dissolved in $0.01 \%$ hydrochloric acid methanol and stored at $4^{\circ} \mathrm{C}$. The standard solution of benzoylaconitine, benzoylmesaconine, and benzoylhypacoitine was diluted to a concentration gradient of $0.05,0.1,0.2,0.4,0.8$, and $1 \mathrm{mg} / \mathrm{mL}$. All final solutions were filtered through a $0.45 \mu \mathrm{m}$ filter membrane, and $20 \mu \mathrm{L}$ filtrate was injected into the HPLC system for analysis.

2.2. HPLC-DAD Analysis. Chromatographic separation was performed on a ChromCore PFP column $(5 \mu \mathrm{m}$, $4.6 \times 250 \mathrm{~mm}$ ) keeping at $30^{\circ} \mathrm{C}$. The flow was $1.0 \mathrm{~mL} / \mathrm{min}$. The UV wave to determinate benzoylaconitine, benzoylmesaconine, and benzoylhypacoitine was $235 \mathrm{~nm}$. The analysis of mobile phase consisting of acetonitrile $(A)$ and $0.04 \mathrm{~mol} / \mathrm{L}$ ammonium acetate (B) was applied to gradient elute: $0 \mathrm{~min}, 35 \% \mathrm{~A} ; 5 \mathrm{~min}, 40 \% \mathrm{~A} ; 35 \mathrm{~min}, 50 \% \mathrm{~A}$.

2.3. Reagents and Antibodies. Complete Freund's adjuvant was supplied from Sigma Company (St Louis, MO, USA). Methotrexate (MTX) was manufactured by SPH Sine Pharmaceutical Laboratories Co., Ltd. (Shanghai, China) (Number: 036180803). Interleukin (IL)-1 $\beta$ and IL-17A enzyme-linked immunosorbent assay (ELISA) kits were purchased from Multi Sciences Biotechnology Co., Ltd. (Zhejiang, China). Primary antibodies against COX-1 and COX-2 were supplied from Multi Sciences Biotechnology Co., Ltd. (Zhejiang, China). 
2.4. Animals. 5-6-week male-specific pathogen-free Wistar rats of level weighing $180 \pm 20 \mathrm{~g}$ were purchased from Chengdu Dashuo Laboratory Experimental Animal Technology Co., Ltd. (Chengdu, China, animal certification number: SCXK (Chuan) 2015-030). The rats were in metal cages with a $12 \mathrm{~h}: 12 \mathrm{~h}$ light-dark cycle in temperaturecontrolled environment $\left(22 \pm 1^{\circ} \mathrm{C}, 55 \pm 5 \%\right.$ relative humidity) for 1 week before the experiment. All animals and procedures in this study were approved by the Ethics Committee of Chengdu University of Traditional Chinese Medicine.

2.5. Clinical Evaluation of AIA Rats. The body weights of rats were gauged at day 7 intervals. The right hind paw volumes (HPV) were assessed via the water displacement method with a plethysmometer on days $0,7,14,21,28$, and 35 until day 42 as primary swelling.

2.6. CFA-Induced Chronic Inflammatory Pain. 36 rats were divided randomly into 6 groups $(n=6)$ : (1) control, (2) AIA (3) MTX, (4) low dose AC (150 mg/kg), (5) middle dose AC (300 mg/kg), and (6) high-dose AC (600 mg/kg) groups. The MTX group was used as the positive control group. The arthritis syndrome of groups (2)-(6) was induced by the intradermal injection with CFA, containing $10 \mathrm{mg}$ heatinactive BCG in $1 \mathrm{~mL}$ paraffin oil, into the right hind paw in $0.1 \mathrm{~mL}$ for each rat and followed by a booster immunization on day 28 [23]. The control group received an intradermal injection of $0.1 \mathrm{~mL}$ of saline throughout the 42-day experiment.

AIA rats were induced at day 0 and drugs were administered from day 14; then photographic images were taken on day 42, at which point the study was ended. Control group and AIA group rats received normal saline $(1 \mathrm{~mL} /$ $100 \mathrm{~g}$ ) by oral gavage. Each AC (low, middle, and high) group's rats received AC solution (dose: 150, 300, and $600 \mathrm{mg} / \mathrm{kg}$ ) by intragastric administration. On day 42 , a photo of the right hind paw of the rats was taken; then, all rats were sacrificed after anesthesia. The immune organs and synovial tissues of each rat were collected and weighed. Hind paws samples were collected and then stored at $4 \%$ paraformaldehyde.

2.7. Indexes of Immune Organs. To assess the effect of AC on immune organs in AIA rats, the indexes of the spleen and thymus were measured. Immune organ index was expressed as the organ weight (mg) versus body weight (g) [24]. Spleen and thymus indexes were calculated according to the following formula:

Spleen index $(\mathrm{mg} / \mathrm{g})=$ spleen weight $(\mathrm{mg}) /$ animal body weight $(\mathrm{g})$

Thymus index $(\mathrm{mg} / \mathrm{g})=$ thymus weight $(\mathrm{mg}) / \mathrm{animal}$ body weight $(\mathrm{g})$

2.8. Hematoxylin and Eosin (HळE) Staining. The rats were sacrificed on day 42 , and the knee joint samples were collected and then stored at $4 \%$ paraformaldehyde. The synovial tissue preparation and H\&E staining were performed. The slides were examined using the Nikon Eclipse E100 Normal phase microscope (Japan), shooting with Nikon digital sight DS-FI2 imaging system (Japan).

\subsection{Measurement of $I L-1 \beta$ and $I L-17 A$ Levels in the Serum.} The rats were anesthetized by using $10 \%$ chloral hydrate $(1 \mathrm{~mL} / 200 \mathrm{~g})$ on day 42 . The blood sample was gathered from the retroorbital vein puncture and then centrifuged at $3500 \mathrm{rpm}$ for $15 \mathrm{~min}$ to obtain the serum. IL- $1 \beta$ and IL-17A in the serum were detected by ELISA kits at $490 \mathrm{~nm}$ UV wave in a microplate reader (Thermo, USA).

2.10. Western Blotting Assay. After the rats were sacrificed on day 42, the synovial tissues were separated from the knee joints of rats. The expression levels of COX-1 and COX-2 proteins in synovial tissues were assessed using Western blot. Synovial tissue was lysed with ice-cold RIPA buffer, the insoluble materials were removed, and protein concentrations of samples were measured using Protein Assay Kit (Zhejiang, China). The proteins were separated by $10 \%$ sodium dodecyl sulfate-polyacrylamide gel electrophoresis and transferred onto polyvinylidene difluoride membranes. After blocking the blots with 5\% skimmed milk powder, the membranes were then incubated with anti-COX-1 $(1: 3000$, rabbit polyclonal (ab51398-050), Zhejiang, China), antiCOX-2 (1:3000, rabbit polyclonal, Zhejiang, China), and anti- $\beta$-actin (1:3000, mouse polyclonal (ab8863-050), Zhejiang, China) overnight at $4^{\circ} \mathrm{C}$. Subsequently, the membrane was washed with TBST and incubated with the secondary antibodies at room temperature. The bands were detected by the electrochemiluminescence detection method. The immunoblot intensity was quantified using Alpha software.

2.11. RNA Isolation and Quantitative Real-Time PCR ( $q R T$ $P C R)$. After the rats were sacrificed on day 42, the synovial tissues were separated from the knee joints of rats. The gene expression levels of COX-1 and COX-2 in synovial tissues were assessed using qRT-PCR. RNA in synovial tissue was isolated using the TriPure Isolation Reagent. The first strand cDNA was reversely transcribed and used as the DNA templates for analysis of gene mRNA relative expression levels. qRT-PCR reactions were conducted on a CFX96 RealTime PCR system with the Transcriptor First Strand cDNA Synthesis Kit (GHA). PCR reaction was set as follows: $95^{\circ} \mathrm{C}$ for $2 \mathrm{~min}, 95^{\circ} \mathrm{C}$ for $10 \mathrm{~s}, 40$ cycles of $60^{\circ} \mathrm{C}$ for $30 \mathrm{~s}$, and $95^{\circ} \mathrm{C}$ for $15 \mathrm{~s}$. It was performed in triplicate for each sample and the relative expression level was expressed using $2^{-\triangle \Delta \mathrm{Ct}}$ method (Table 1).

2.12. Statistical Analysis. All statistical analyses were calculated by using one-way ANOVA followed by the post hoc Dunnett's test, which were applied to evaluate the significant differences among the groups. Data were presented as mean \pm standard deviation (SD) and $P<0.05$ was considered 
TABle 1: Primer pair sequences used for qRT-PCR.

\begin{tabular}{lcc}
\hline Genes & Forward & Reverse \\
\hline COX-1 & CAGGAGGTGTTTGGGTTGCT & CTATAAGGATGAGGCGAGTGGT \\
COX-2 & ACTACGCCTGAGTTTCTGACAAG & TTCACAATGTTCCAGACTCCCT \\
$\beta$-Actin & TGTCACCAACTGGGACGATA & GGGGTGTTGAAGGTCTCAAA \\
\hline
\end{tabular}

to be statistically significant. All data were analyzed by the SPSS 17.0 software.

\section{Results and Discussion}

3.1. HPLC-DAD Analysis of AC. Three chemicals were quantified by calculating the peak area, and they were identified by comparing the retention times of each peak with the authentic references. The contents of the three main chemicals were found to be $0.084 \%$ for benzoylaconitine, $0.0276 \%$ for benzoylmesaconine, and $0.004 \%$ for benzoylhypacoitine (Figure 1).

3.2. Clinical Evaluation of AIA Rats. As shown in Figure 2, the redness and swelling of the right hind paw volume (HPV) of rats in the AIA group were markedly increased compared with the rats in the control group. Following AC treatment with 300 and $600 \mathrm{mg} / \mathrm{kg}$ since day 14, paw swellings were then markedly reduced compared to the AIA group.

HPV at days $0,7,14,21,28,35$, and 42 was evaluated in terms of swelling severity by complete Freund's adjuvant(CFA-) induced. As shown in Figure 3(a), the HPV of the model group initially increased and reached its maximum value on day 21. Then, it decreased slowly from day 21 to 28 . After booster immunization on day 28, the HPV slowly started to increase from day 35. The entire paw swelling process in the AIA group emulated the state of arthritis. Figure 3(a) shows that the HPV of the AIA group was markedly increased compared with the control group. In contrast, the swelling of rats in the treatment groups was mitigated, and AC could alleviate paw swelling from day 14 . On day 21 , a slight decrease in AC $(600 \mathrm{mg} / \mathrm{kg})$ was observed compared with the AIA group. Between days 28 and 42, rats were treated with $300 \mathrm{mg} / \mathrm{kg}$ and $600 \mathrm{mg} / \mathrm{kg}$ of $\mathrm{AC}$ $(P<0.01)$, and they exhibited a significantly lower HPV compared to AIA rats, which were dose-dependent. Figure 3(b) shows the results of body weight, which was used to evaluate AC improvement on the holistic health of AIA rats. Compared with the control group, all other groups exhibited a significant loss in their body weight.

3.3. Effects of AC on the Immune Organ Index. Spleen and thymus are important immune organs, and the organ index can reflect the strength of the immune function. The immune organ index is shown in Figures 4(a) and 4(b). Compared with the control group, CFA-induced rats showed significant enlargement on spleen and thymus tissues. After treatment with $\mathrm{AC}$, the spleen index of the AC $(150,300$, and $600 \mathrm{mg} / \mathrm{kg})$ group was significantly reduced compared with the AIA group. Similarly, the thymus index of the AC $(600 \mathrm{mg} / \mathrm{kg})$ group also showed a significant reduction compared with the AIA group.

3.4. AC Restores Histological Changes of the Synovial Tissue in AIA Rats. The effects of AC on cartilage damage and synovial hyperplasia in the knee joint of AIA rats were evaluated by histopathological studies. As shown in Figure 5, the knee joints in the AIA group showed severe synovial tissue proliferation, inflammatory cell infiltration, and cartilage and bone erosion compared to the control group. The AC $(150 \mathrm{mg} / \mathrm{kg})$ group revealed significant inflammatory cells infiltration in the synovial membrane. In contrast, mild inflammatory cells infiltration was observed in the MTX and AC (300 and $600 \mathrm{mg} / \mathrm{kg}$ ) groups, which also demonstrated normal histological structure. This finding suggests that MTX and AC (300 and $600 \mathrm{mg} / \mathrm{kg}$ ) could reverse CFA-induced histopathological alterations in the synovial membrane, articular cartilaginous surface, and bony structure.

3.5. Effects of AC on IL-1 $\beta$ and IL-17A Levels. As shown in Figures $6(\mathrm{a})$ and $6(\mathrm{~b})$, IL-1 $\beta$ and IL-17A were measured in order to assess the anti-inflammation effect of AC. IL- $1 \beta$ and IL-17A levels were found to be significantly increased in the AIA group compared to the control group $(P<0.01)$. The expressions of IL- $1 \beta$ in the AC $(600 \mathrm{mg} / \mathrm{kg})$ and MTX group were significantly reduced compared to the AIA group. IL$17 \mathrm{~A}$ production after $\mathrm{AC}(150,300$, and $600 \mathrm{mg} / \mathrm{kg})$ and MTX group administration were also markedly reduced compared to the AIA group. Therefore, AC can obviously inhibit inflammatory cytokines in the serum of AIA rats.

3.6. Effects of AC on COX-1 and COX-2 Protein Expression. To further investigate the anti-inflammatory mechanism of AC on CFA-induced adjuvant arthritis in rats, we detected the inflammation-related signaling pathway of cyclooxygenase. As shown in Figure 7, the AIA group showed significant elevation in both COX-1 and COX-2 levels compared with the control group $(P<0.01)$, whereas AC could effectively downregulate the protein levels of COX-1 and COX-2. The AC (300 and $600 \mathrm{mg} / \mathrm{kg}$ ) groups revealed a significant decrease in COX-1 and COX-2 compared with the AIA group $(P<0.05 ; P<0.01)$. Among the three doses of AC groups, $600 \mathrm{mg} / \mathrm{kg}$ exhibited the strongest effect $(P<0.01)$, and there was no significant difference compared with the control group.

3.7. Effects of $A C$ on $C O X-1$ and $C O X-2$ Gene Expression. To further substantiate the above findings, we measured the mRNA levels of COX-1 (Figure 8(a)) and COX-2 


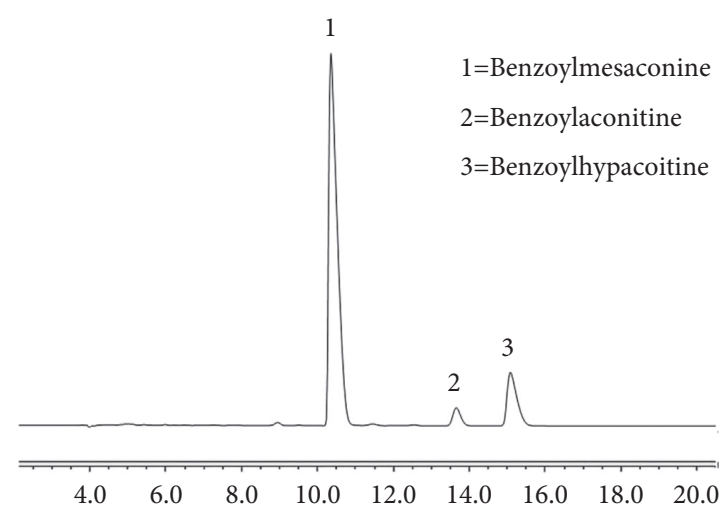

(a)
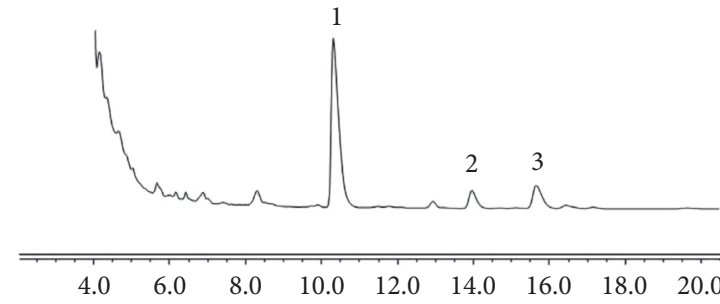

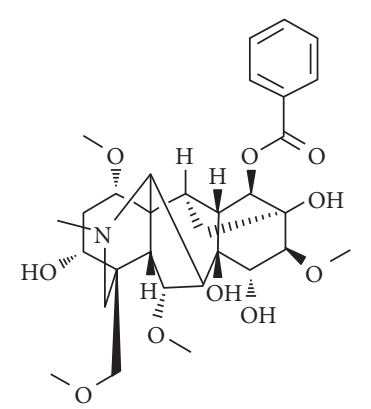

1

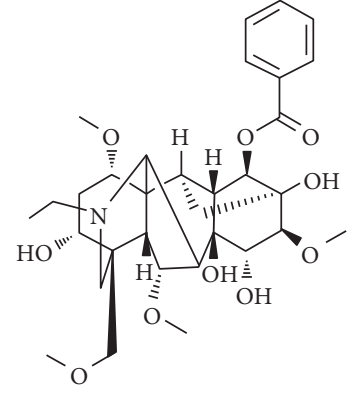

2

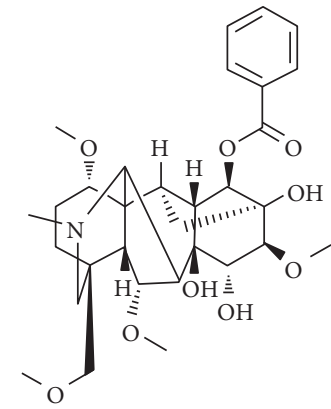

3

(b)

Figure 1: Chromatograms of (a) reference standards and (b) AC decoction.

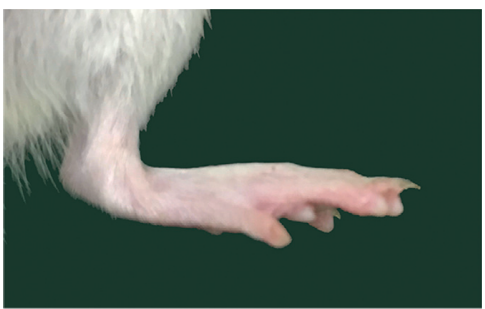

Control

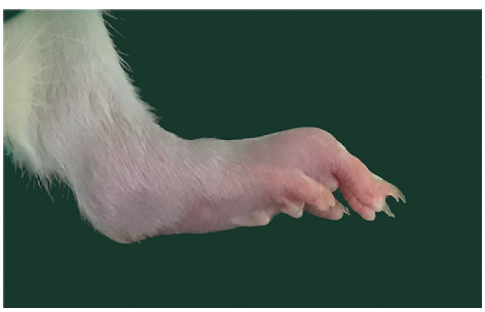

AC $(150 \mathrm{mg} / \mathrm{kg})$

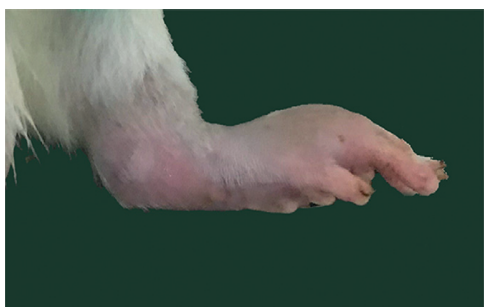

AIA

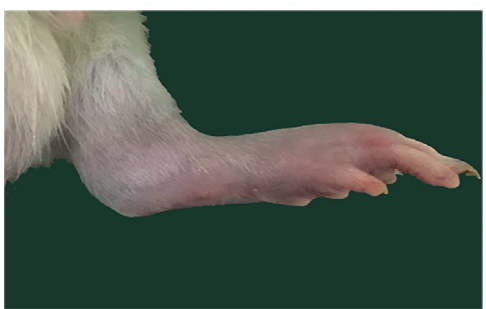

AC $(300 \mathrm{mg} / \mathrm{kg})$

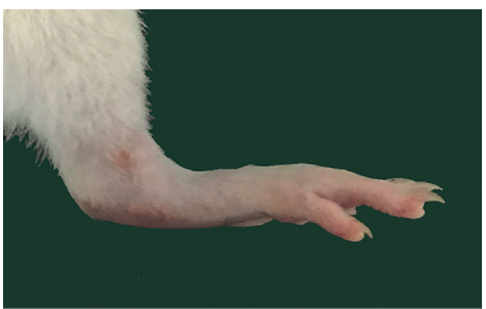

MTX

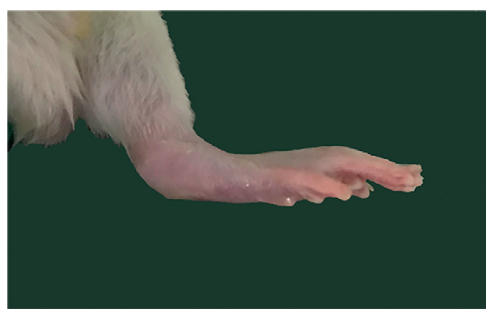

AC $(600 \mathrm{mg} / \mathrm{kg})$

FIGURE 2: Representative photos of paw swelling of each group on the 42nd day. 


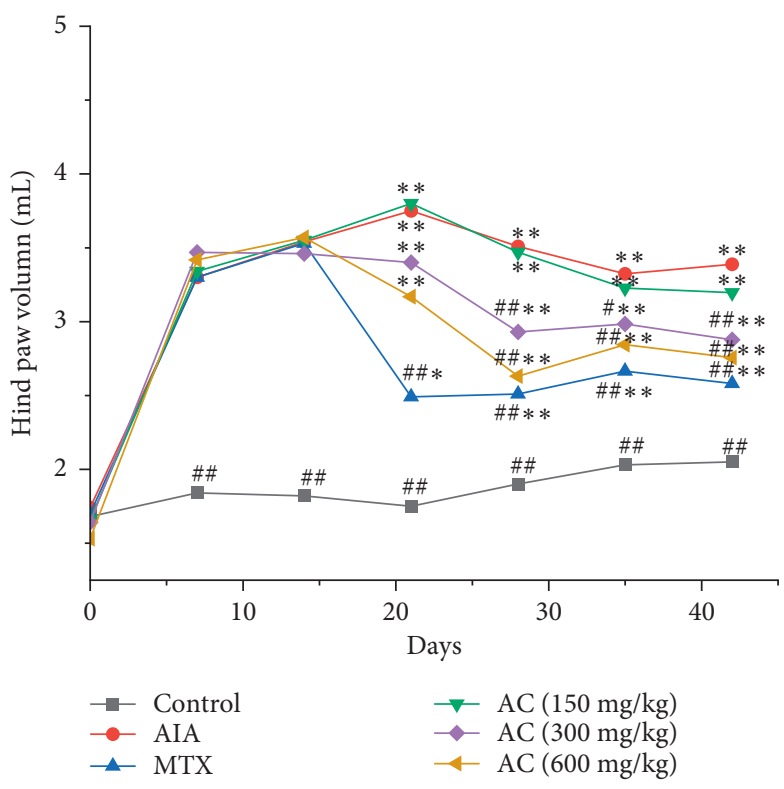

(a)

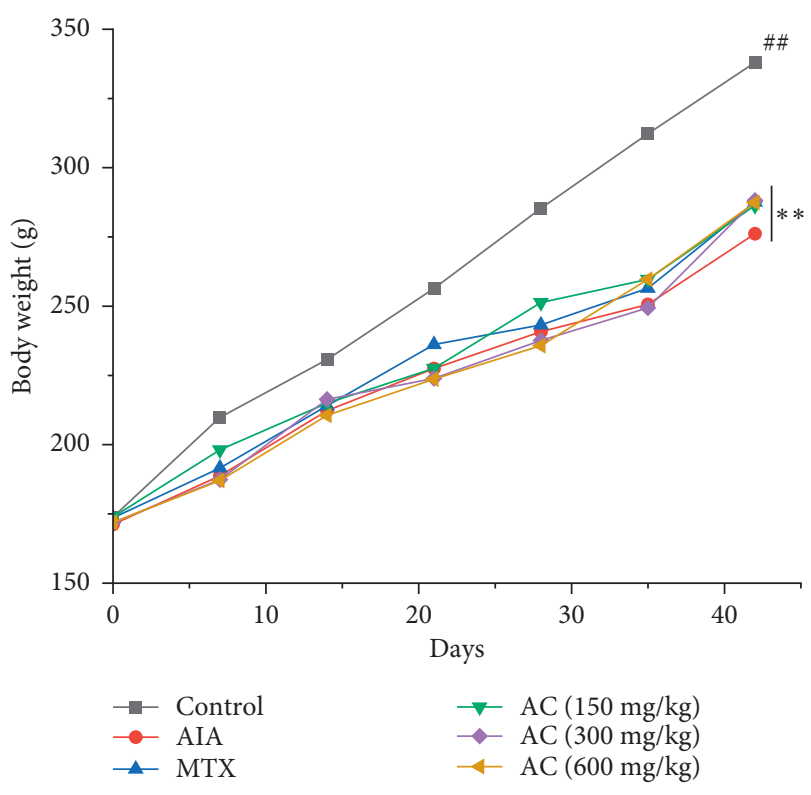

(b)

Figure 3: (a) Hind paw volume during 42 days between the AIA and the AC treatment. (b) Effect of AC on body weight. ${ }^{*} P<0.05$ and ${ }^{* *} P<0.01$, compared with the control group; ${ }^{\#} P<0.05$ and ${ }^{\# \#} P<0.01$, compared with the AIA group $(n=6)$.

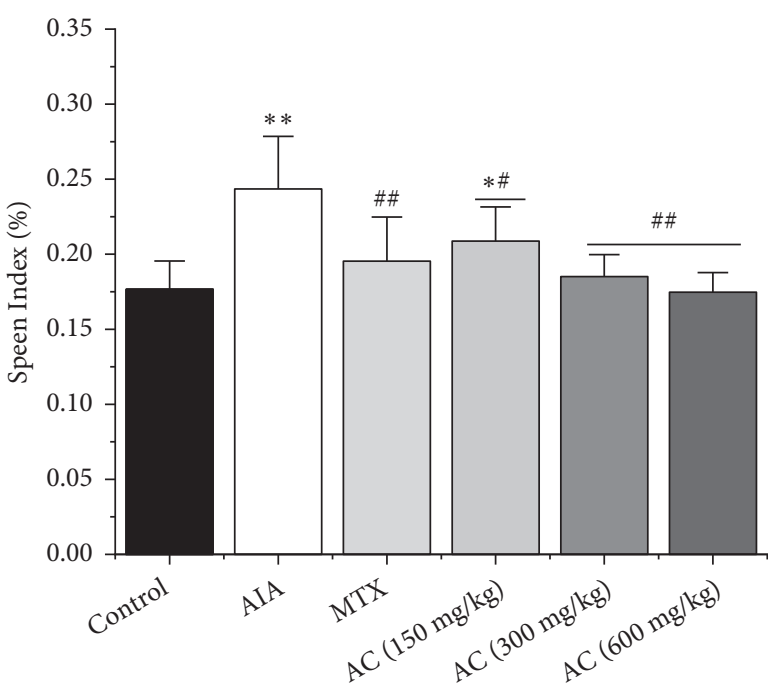

(a)

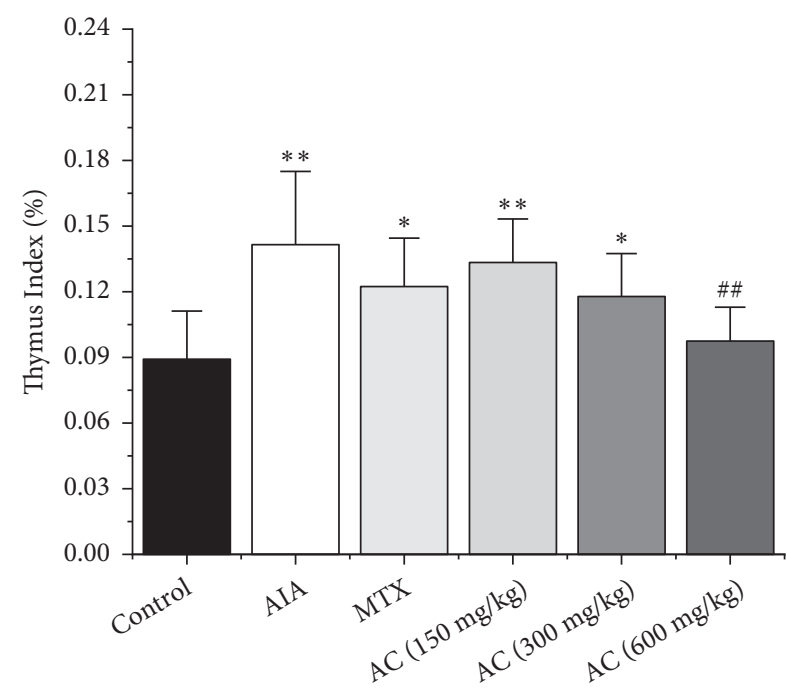

(b)

Figure 4: Immune organ index in each group. (a) Indexes of the spleen. (b) Indexes of the thymus. ${ }^{*} P<0.05$ and ${ }^{* *} P<0.01$, compared with the control group; ${ }^{\#} P<0.05$ and ${ }^{\# \#} P<0.01$, compared with the AIA group $(n=6)$.

(Figure $8(\mathrm{~b})$ ) in synovial tissues by real-time quantitative reverse transcription polymerase chain reaction (qRTPCR) to evaluate the effects of AC at the transcriptional level, and the respective findings are shown in Figures 8(a) and $8(\mathrm{~b})$. COX-1 mRNA expression in the AIA group rats remained unchanged compared to the control group $(P>0.05)$. A tendency of elevated COX-1 mRNA levels by the AC (150 and $300 \mathrm{mg} / \mathrm{kg}$ ) groups was found, which was nonsignificant. Although the AC $(600 \mathrm{mg} / \mathrm{kg})$ group can reduce the COX-1 mRNA levels, it did not reveal statistically significant differences with the control group and AIA group $(P>0.05)$. Furthermore, COX-2 mRNA was significantly upregulated in the AIA group compared with the control group $(P<0.01)$. The mRNA levels of COX-2 in the AC $(150,300$, and $600 \mathrm{mg} / \mathrm{kg})$-treated groups were significantly reduced compared with the AIA group $(P<0.01)$. According to the qRT-PCR results, the AC (150, 300 , and $600 \mathrm{mg} / \mathrm{kg}$ ) groups exhibited clear effects on reducing the mRNA levels of COX-2 in synovial tissues. However, the AC group did not induce any significant 


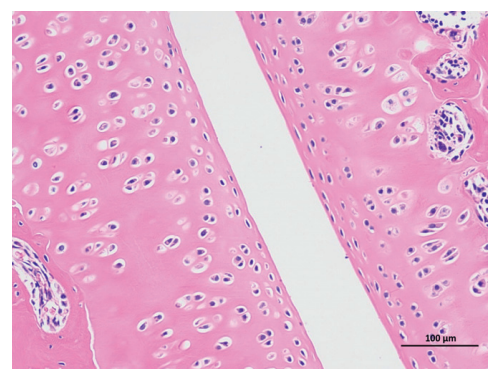

(a)

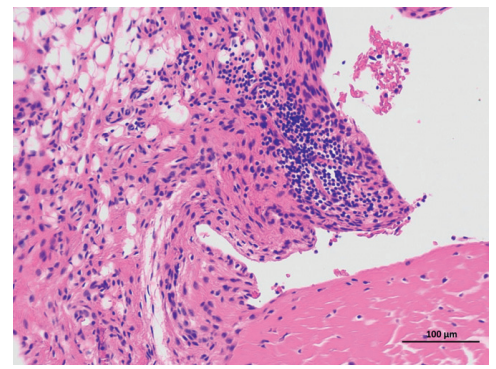

(d)

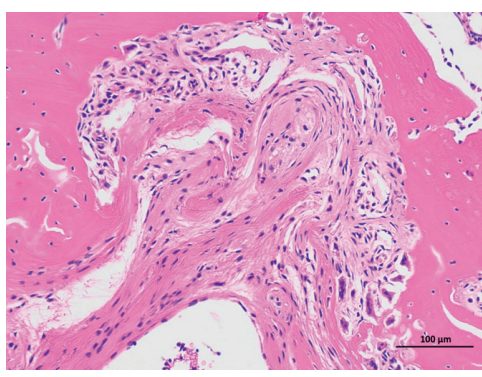

(b)

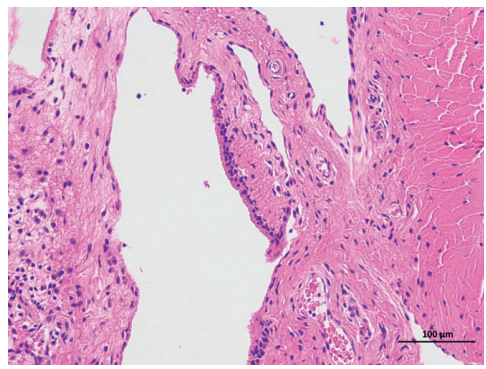

(e)

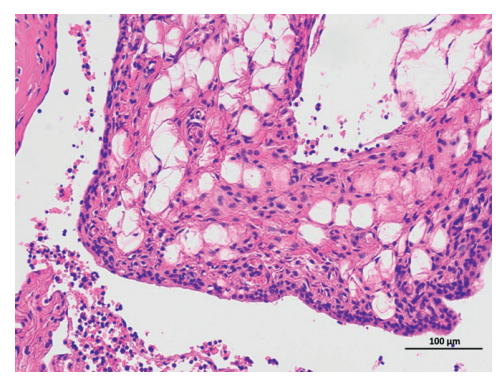

(c)

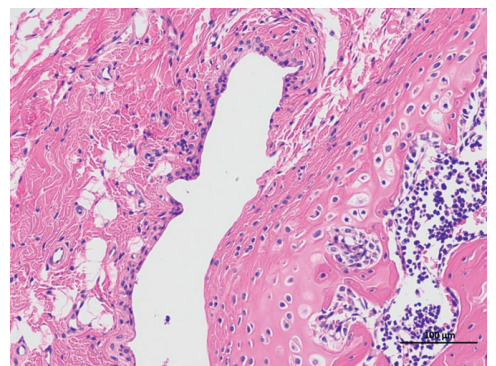

(f)

Figure 5: Histopathology of the right hind knee joint in each group (x 200, scale bars = $100 \mu \mathrm{m}$ ). (a) Control; (b) AIA; (c) MTX; (d) AC (150 mg/kg); (e) AC (300 mg/kg); (f) AC (600 mg/kg).

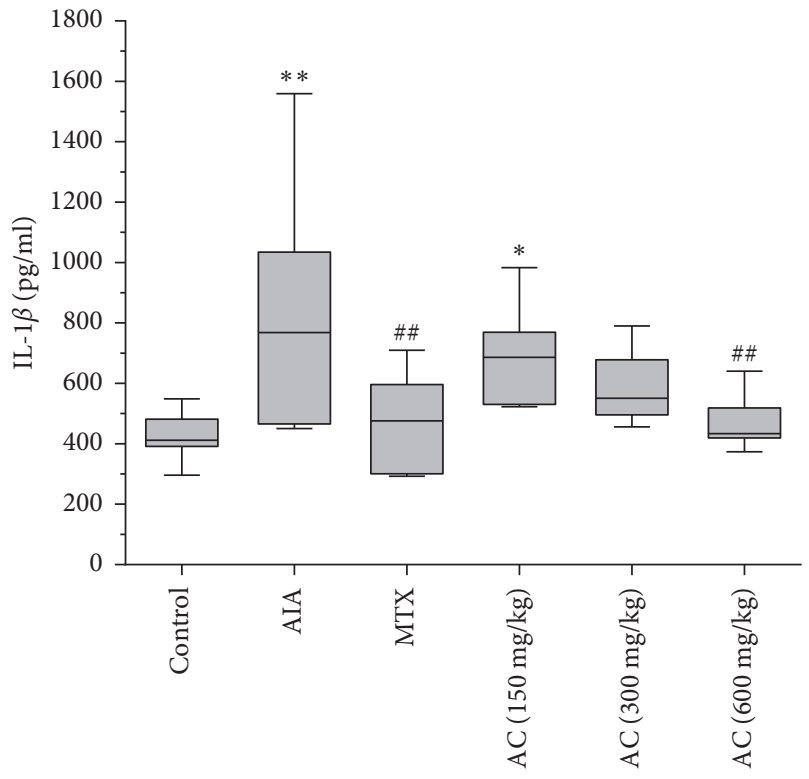

(a)

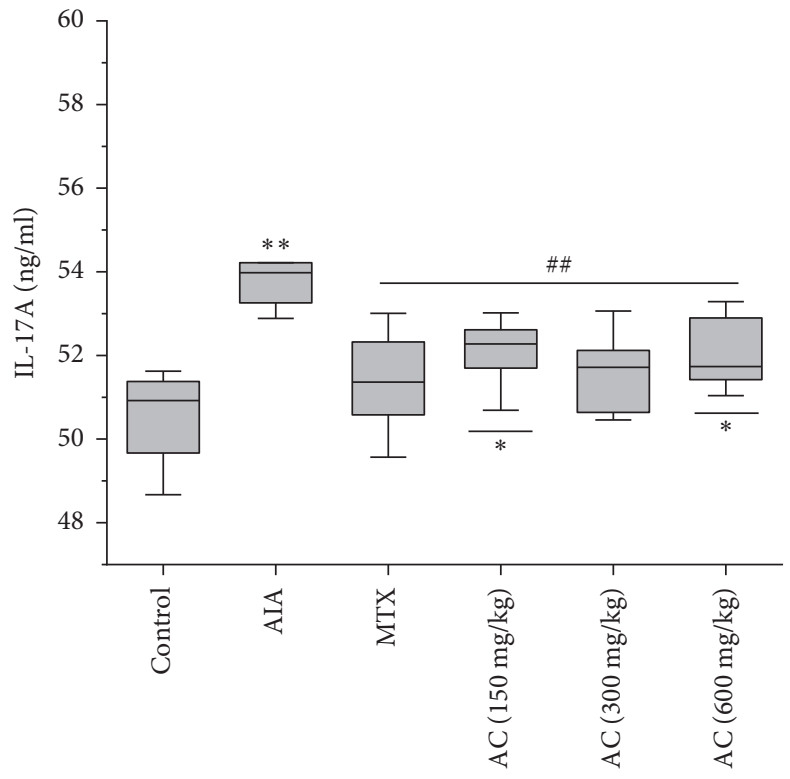

(b)

Figure 6: Effects of AC on IL-1 $\beta$ and IL-17A levels in serum via ELISA. ${ }^{*} P<0.05$ and ${ }^{* *} P<0.01$, compared with the control group; ${ }^{\#} P<0.05$ and ${ }^{\# \#} P<0.01$, compared with the AIA group $(n=6)$.

effect on COX-1 mRNA expression in RA rats' synovial tissues.

\section{Discussion}

AIA is a commonly used method to assess the antiarthritic effect of various agents, with many of the clinical and pathological characteristics found in human RA condition.
We employed the AIA rat model to evaluate the antiarthritic activity of AC. Intradermal injection of CFA could induce infiltration of the synovial membrane, production of proinflammatory factors, and accumulation of neutrophils, resulting in persistent nociceptive stimulation. AIA induced significant inflammatory (lymphocytes) cell infiltration, synovial hyperplasia, cartilage erosion, and pannus formation, as shown in the respective histopathological images. 


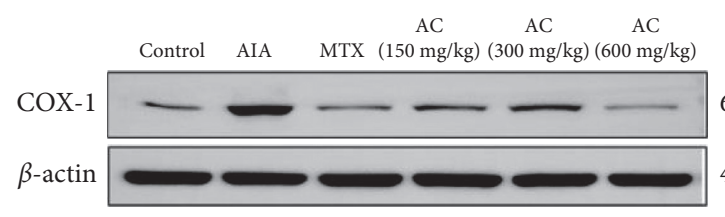

(a)

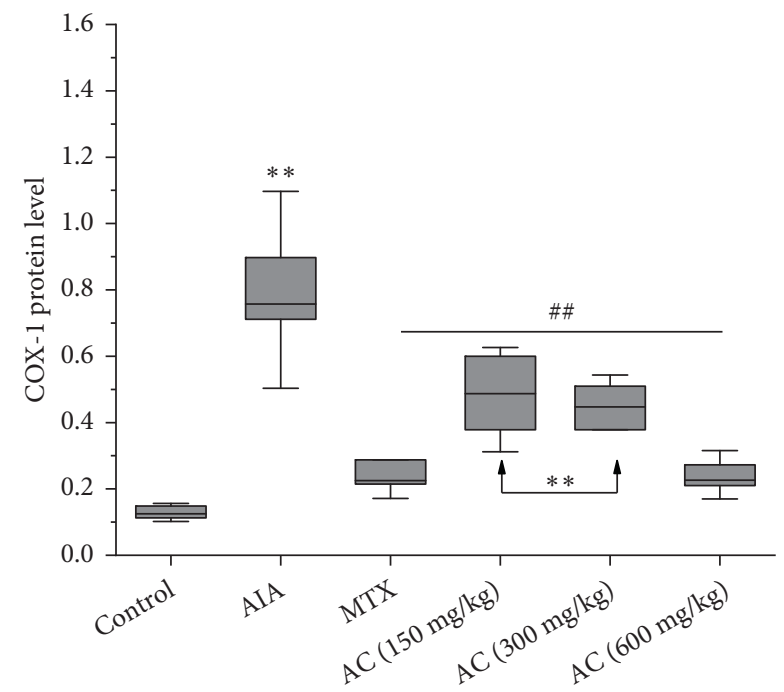

(c)

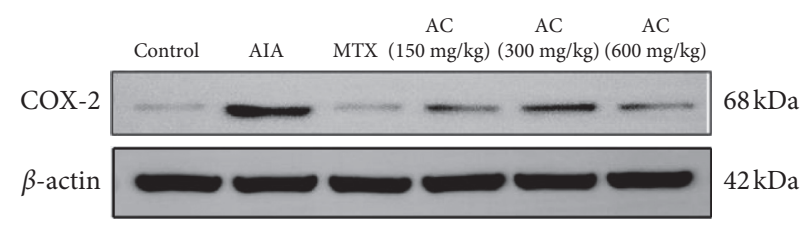

(b)

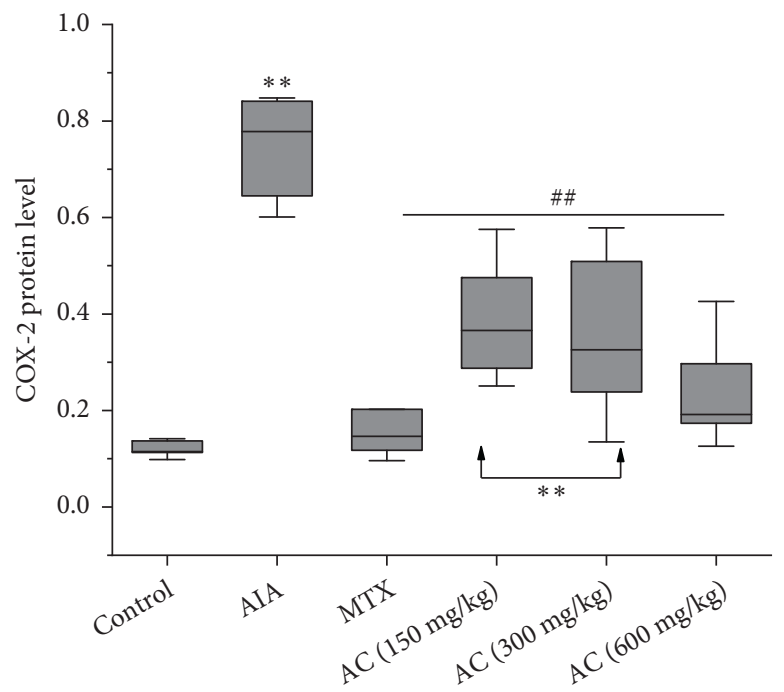

(d)

Figure 7: (a) Protein expression of COX-1 in the synovial tissues. (b) Protein expression of COX-2 in the synovial tissues. (c) Western blot analysis of the expressed level of COX-1 in the synovial tissues. (d) Western blot analysis of the expressed level of COX-2 in the synovial tissues. ${ }^{*} P<0.05$ and ${ }^{* *} P<0.01$, compared with the control group. ${ }^{\#} P<0.05$ and ${ }^{\# \#} P<0.01$, compared with the AIA group ( $\left.n=6\right)$.

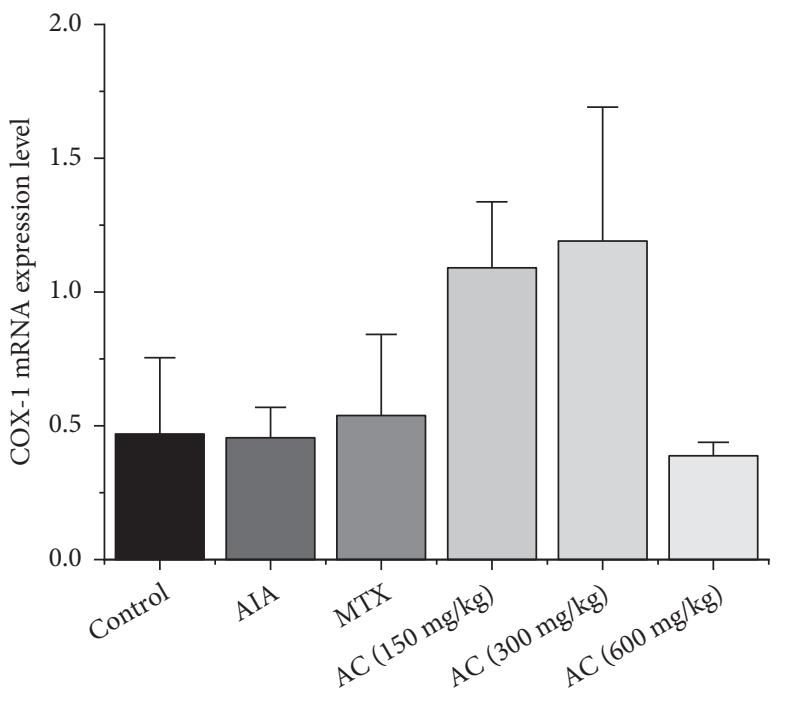

(a)

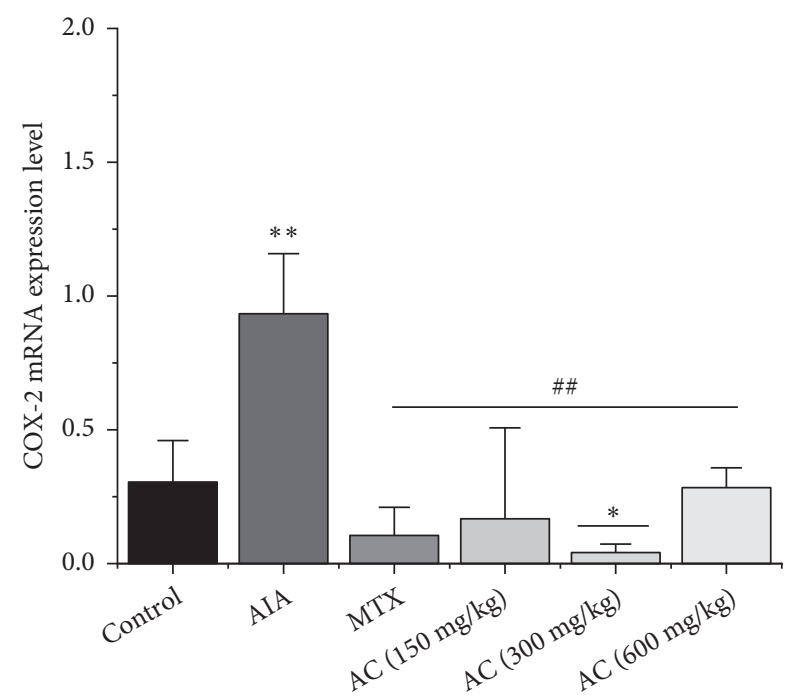

(b)

FIGURE 8: (a) qRT-PCR analysis of the expressed level was presented of COX-1 in the synovial tissues; (b) qRT-PCR analysis of the expressed level was presented of COX-2 in the synovial tissues. ${ }^{*} P<0.05$ and ${ }^{* *} P<0.01$, compared with the control group. ${ }^{\#} P<0.05$ and ${ }^{\# \#} P<0.01$, compared with the AIA group $(n=6)$.

Additionally, AIA significantly increased the levels of IL- $1 \beta$, IL-17A in serum. Chronic inflammation in the AIA model was mainly manifested as a progressive increase in the volume of the injected paw. Moreover, the arthritic rats lost weight compared with the control group. These results demonstrate the success of the arthritis model employed.

The metabolic and immunomodulatory activity of different drugs is typically evaluated based on both body weight 
and immune organ index values in treated animals [25]. CFA induced can lead to profound immunosuppression and to a reduction in rat body weight caused by promoting the production of macrophages and lymphocytes [26]. Spleen and thymus weights relate to the number of immune cells, and they both play an important role in cellular immunity by serving as reservoirs for antibody storage [27]. In this study, the spleen and thymus indexes of the AC (300 and $600 \mathrm{mg}$ / $\mathrm{kg}$ ) groups significantly decreased, which is possibly caused by a reduction in AC lymphocyte proliferation. The results of the organ index showed that AC could enhance the body's immune function.

MTX could greatly alleviate the swelling of the rats' hind paw in the first 14 days, thus indicating a quick effect. This may be related to the direct anti-inflammatory effect and inhibition of leukocyte tropism of MTX. However, MTX exerted its weakened pharmacological effect after booster immunization, when, at the same time, AC gradually exerted its beneficial effects. AC was found to greatly inhibit the development of the disease. This suggests that AC has a lasting therapeutic effect on RA. At the initial stage of AC administration, a slight reduction in the paw swelling of rats was observed, which accumulated with the administration time. AC gradually exerted its efficacy at a later stage, and its efficacy was equal to the efficacy of MTX. Therefore, we speculate that the efficacy of AC may be attributed to a cumulative effect.

RA is characterized by persistent inflammatory synovitis. Proinflammatory cytokines play a vital role in the pathogenesis of synovitis and articular destruction in RA. Thus, they are both important targets for RA treatment $[28,29]$. Interleukin- $\beta$ (IL-1 $\beta$ ) and interleukin-17A (IL-17A), as proinflammatory cytokines, have been reported to be significantly expressed in the rheumatoid joint [30], which could lead to joint synovial destruction. IL-1 $\beta$, the predominant extracellular form of IL-1, has been regarded as an important proinflammatory cytokine in the pathogenesis of RA. It is produced at the early stages of the immune response, with a strong proinflammatory effect, which mainly causes bone and cartilage destruction [31]. IL-17A also plays a crucial role in RA. It is mainly produced by T cells and leads to osteoclasts activation and to the increased production of other cytokines $[32,33]$. In the AIA model, activation and release of proinflammatory cytokines and interleukins were found to alter osteoclast differentiation, leading to bone erosion [34]. Histopathological images of synovial tissues revealed the pronounced cell infiltration of lymphocytes accompanied by bone erosion and angiogenesis. In this study, AC reduced the concentrations of inflammatory cytokines IL-1 $\beta$ and IL-17A. In addition, AC not only suppressed inflammatory responses but also alleviated bone erosion and neovascularization in AIA rats. Consequently, AC may be a promising candidate drug for RA treatment.

Cyclooxygenase isoenzymes (COX-1 and COX-2) have a critical role in inflammation through arachidonic acid metabolism pathways. COX-1 is constitutively expressed in the majority of normal tissues for maintaining normal physiological effects, whereas COX-2 is specifically expressed in inflamed tissues and it is unregulated [35, 36]. COX-2 participates in the generation of proinflammatory factors and cartilage destruction, which plays a major role in inflammation and autoimmune disease [37]. Crofford et al. [38] reported that IL- $1 \beta$ could enhance COX-2, mRNA, and protein synthesis in rheumatoid synovial cells but had no effect on COX-1. Early studies had stated that the severe gastrointestinal toxicity of NSAID is due to its inhibitory effect on COX-1 [39]. However, a recent research has shown that selective inhibition of COX-1 activity can effectively treat inflammation without causing gastric damage and cardiovascular disease [40]. Therefore, it is essential to conduct further studies to elucidate whether inhibition of COX-1 can treat RA.

In this study, the effects of $\mathrm{AC}$ on COX-1, COX-2 mRNA, and protein levels in AIA rat synovial tissues were also investigated. Our findings suggest that COX-1 and COX-2 expression were significantly suppressed by AC in translation level. The results suggest that inhibition of COX catalyzed the conversion of arachidonic acid into prostaglandins, and this may be the mechanism by which AC mediates its anti-inflammatory activity. However, we found that COX-1 mRNA level and protein expression are inconsistent. This may be due to the fact that there are three mRNA transcripts in cyclooxygenase: $2.8 \mathrm{~kb}, 4.5 \mathrm{~kb}$, and $5.2 \mathrm{~kb}$. The $2.8 \mathrm{~kb}$ transcript is the most frequently detected because it probably encodes the primary protein form for COX-1 [41]. The COX-1 mRNA level that we detected and which may represent the total mRNA level remained unchanged in the AIA group. However, there may also be additional factors that play a role in this process, such as the effect of posttranslational modification or the fact that the protein was changed from an inactive to an active state by an activating factor. There was no positive correlation between this change and the change in the COX-1 transcription level. The levels of COX-1 mRNA in the synovial tissue of the AIA rats remained unchanged, whereas the level of COX-1 protein increased, which may reveal that regulation of translation level may play a leading role in this process. We also found that the COX-1 mRNA level in the AIA group was not significantly different compared to the control group. However, the mRNA level of COX-1 was increased in the AC $(150 \mathrm{mg} / \mathrm{kg}$ and $300 \mathrm{mg} / \mathrm{kg})$ groups. Although it is generally known that COX-1 limits inflammatory responses, mild modifications can occur in the expression of the enzyme in cells and tissues when they are stimulated by proinflammatory cytokines or injury [42]. Therefore, the regulatory effect of RA on $\mathrm{AC}$ in $\mathrm{COX}-1$ transcription level needs further study.

\section{Conclusion}

In summary, our study indicates that AC is significantly against adjuvant-induced arthritis in rats, by inhibiting immune organs (spleen and thymus), attenuating paw swelling, infiltration of inflammatory cells and synovial hyperplasia, and downregulating the levels of IL- $1 \beta$ and IL$17 \mathrm{~A}$ in AIA rats. In addition, we identified that AC has significant anti-inflammatory therapeutic effects. Further molecular mechanism is to regulate arachidonic acid 
metabolism pathways. Particularly, AC could ameliorate overexpression of COX-2. Although these results show that the use of $\mathrm{AC}$ for RA treatment can be very promising, further research should be carried out to elucidate in greater detail the molecular mechanisms involved in these antiarthritic properties of AC.

\section{Data Availability}

The data used to support the findings of this study are included within the article.

\section{Conflicts of Interest}

The authors declare that they have no conflicts of interest.

\section{Acknowledgments}

This work was supported by the National Natural Science Foundation of China (81703825), the Natural Science Foundation Project of the Education Department of Sichuan Province (18ZB01869), and the Sichuan Science and Technology Program (2021YJ0254).

\section{References}

[1] M. Abou-ElNour, R. A. H. Ishak, M. Tiboni et al., "Triamcinolone acetonide-loaded PLA/PEG-PDL microparticles for effective intra-articular delivery: synthesis, optimization, in vitro and in vivo evaluation," Journal of Controlled Release, vol. 309, pp. 125-144, 2019.

[2] F. Matcham, I. C. Scott, L. Rayner et al., "The impact of rheumatoid arthritis on quality-of-life assessed using the SF36: a systematic review and meta-analysis," Seminars in Arthritis and Rheumatism, vol. 44, no. 2, pp. 123-130, 2014.

[3] H. Radner, J. S. Smolen, and D. Aletaha, "Comorbidity affects all domains of physical function and quality of life in patients with rheumatoid arthritis," Rheumatology, vol. 50, no. 2, pp. 381-388, 2011.

[4] S. Safiri, A. A. Kolahi, D. Hoy et al., "Global, regional and national burden of rheumatoid arthritis 1990-2017: a systematic analysis of the global burden of disease study 2017," Annals of the Rheumatic Diseases, vol. 78, no. 11, pp. 1463-1471, 2019.

[5] M. Choudhary, V. Kumar, P. K. Gupta, and S. Singh, "Antiarthritic activity of Barleria prionitis Linn. leaves in acute and chronic models in Sprague Dawley rats," Bulletin of the Faculty of Pharmacy Cairo University, vol. 52, no. 2, pp. 199-209, 2014.

[6] G. S. Hazlewood, C. Barnabe, G. Tomlinson, D. Marshall, D. Devoe, and C. Bombardier, "Methotrexate monotherapy and methotrexate combination therapy with traditional and biologic disease modifying antirheumatic drugs for rheumatoid arthritis: abridged Cochrane systematic review and network meta-analysis," BMJ, vol. 353, p. i1777, 2016.

[7] S. Tacconelli, A. Bruno, R. Grande, P. Ballerini, and P. Patrignani, "Nonsteroidal anti-inflammatory drugs and cardiovascular safety-translating pharmacological data into clinical readouts," Expert Opinion on Drug Safety, vol. 16, no. 7, pp. 791-807, 2017.

[8] C. Zhang, M. Jiang, and A.-p. Lü, "Evidence-based Chinese medicine for rheumatoid arthritis," Journal of Traditional Chinese Medicine, vol. 31, no. 2, pp. 152-157, 2011.
[9] C. Zhu, Q. Xu, Z. Mao, and N. Lin, "The Chinese medicine Wu-tou decoction relieves neuropathic pain by inhibiting hippocampal microglia activation," Scientific Reports, vol. 8, no. 1, Article ID 12292, 2018.

[10] Y. Zhang, X. Mao, Q. Guo et al., "Pathway of PPAR-gamma coactivators in thermogenesis: a pivotal traditional Chinese medicine-associated target for individualized treatment of rheumatoid arthritis," Oncotarget, vol. 7, no. 13, pp. 15885-15900, 2016.

[11] National Pharmacopoeia Committee, Pharmacopoeia of People's Republic of China. Part 1, C hemical Industry Press, Beijing, China, 2020.

[12] W. Gai, X. Hao, J. Zhao et al., "Delivery of benzoylaconitine using biodegradable nanoparticles to suppress inflammation via regulating NF- $\kappa \mathrm{B}$ signaling," Colloids and Surfaces. B, Biointerfaces, vol. 191, Article ID 110980, 2020.

[13] P. Gu, J.-Q. Li, and R.-S. Tong, "Anti-inflammation and analgesia effects of combination therapy with benzoylaconine and paeoniflorin," The Chinese Journal of Modern Applied Pharmacy, vol. 35, no. 8, pp. 1212-1216, 2018.

[14] X. Xia, B. H. May, A. L. Zhang et al., "Chinese herbal medicines for rheumatoid arthritis: text-mining the classical literature for potentially effective natural products," Evidencebased Complementary and Alternative Medicine: eCAM, vol. 2020, Article ID 7531967, 14 pages, 2020.

[15] S. C. Zheng, X. Y. Yan, J. Chen, S. T. Zhao, and C. B. Wen, "Anti-inflammatory mechanism analysis of antirheumatic Chinese medicinal herb Aconiti Radix based on protein interaction network," China Journal of Chinese Materia Medica, vol. 42, no. 9, pp. 1747-1751, 2017.

[16] X. Li, L. L. Mazaleuskaya, L. L. Ballantyne, H. Meng, G. A. FitzGerald, and C. D. Funk, "Genomic and lipidomic analyses differentiate the compensatory roles of two COX isoforms during systemic inflammation in mice," Journal of Lipid Research, vol. 59, no. 1, pp. 102-112, 2018.

[17] K. Subbaramaiah and A. J. Dannenberg, "Cyclooxygenase-2 transcription is regulated by human papillomavirus 16 E6 and E7 oncoproteins: evidence of a corepressor/coactivator exchange," Cancer Research, vol. 67, no. 8, pp. 3976-3985, 2007.

[18] R. J. Flower, “The development of COX2 inhibitors," Nature Reviews Drug Discovery, vol. 2, no. 3, pp. 179-191, 2003.

[19] R. G. Kurumbail, A. M. Stevens, J. K. Gierse et al., "Structural basis for selective inhibition of cyclooxygenase-2 by antiinflammatory agents," Nature, vol. 384, no. 6610, pp. 644-648, 1996.

[20] C. Park, D.-O. Moon, I.-W. Choi et al., "Curcumin induces apoptosis and inhibits prostaglandin E2 production in synovial fibroblasts of patients with rheumatoid arthritis," International Journal of Molecular Medicine, vol. 20, no. 3, pp. 365-372, 2007.

[21] P. A. Gourraud, P. Dieudé, J. F. Boyer et al., "A new classification of HLA-DRB1 alleles differentiates predisposing and protective alleles for autoantibody production in rheumatoid arthritis," Arthritis Research \& Therapy, vol. 9, no. 2, p. R27, 2007.

[22] N. Choudhary, L. K. Bhatt, and K. S. Prabhavalkar, "Experimental animal models for rheumatoid arthritis," Immunopharmacology and Immunotoxicology, vol. 40, no. 3, pp. 193-200, 2018.

[23] M. Liu, W. Mao, H. Guan, L. Li, B. Wei, and P. Li, "Effects of taurochenodeoxycholic acid on adjuvant arthritis in rats," International Immunopharmacology, vol. 11, no. 12, pp. 2150-2158, 2011. 
[24] X.-X. Zhang, Y. Ito, J.-R. Liang, J.-L. Liu, J. He, and W.-J. Sun, "Therapeutic effects of total steroid saponin extracts from the rhizome of Dioscorea zingiberensis C. H. Wright in Freund's complete adjuvant induced arthritis in rats," International Immunopharmacology, vol. 23, no. 2, pp. 407-416, 2014.

[25] Q. Cao, X. Gao, Y. Lin et al., "Thymopentin ameliorates dextran sulfate sodium-induced colitis by triggering the production of IL-22 in both innate and adaptive lymphocytes," Theranostics, vol. 9, no. 25, pp. 7490-7505, 2019.

[26] M. Carol, C. Pelegrí, C. Castellote, A. Franch, and M. Castell, "Immunohistochemical study of lymphoid tissues in adjuvant arthritis (AA) by image analysis; relationship with synovial lesions," Clinical and Experimental Immunology, vol. 120, no. 1, pp. 200-208, 2000.

[27] R. Sundaram, R. Naresh, P. Shanthi, and P. Sachdanandam, "Antihyperglycemic effect of iridoid glucoside, isolated from the leaves of Vitex negundo in streptozotocin-induced diabetic rats with special reference to glycoprotein components," Phytomedicine, vol. 19, no. 3-4, pp. 211-216, 2012.

[28] N. Eugénia and G. Andreiaa, "Pretoa ana, and C.-P. Artur. "Update on therapeutic approaches for rheumatoid arthritis"” Current Medicinal Chemistry, vol. 23, no. 21, pp. 1-14, 2016.

[29] G. Schett and E. Gravallese, "Bone erosion in rheumatoid arthritis: mechanisms, diagnosis and treatment," Nature Reviews Rheumatology, vol. 8, no. 11, pp. 656-664, 2012.

[30] A. K. Ulfgren, S. Lindblad, L. Klareskog, J. Andersson, and U. Andersson, "Detection of cytokine producing cells in the synovial membrane from patients with rheumatoid arthritis," Annals of the Rheumatic Diseases, vol. 54, no. 8, pp. 654-661, 1995.

[31] G. Arango Duque and A. Descoteaux, "Macrophage cytokines: involvement in immunity and infectious diseases," Frontiers in Immunology, vol. 5, no. 7, p. 491, 2014.

[32] M. Noack and P. Miossec, "Selected cytokine pathways in rheumatoid arthritis," Seminars in Immunopathology, vol. 39, no. 4, pp. 365-383, 2017.

[33] J. Alam, I. Jantan, and S. N. A. Bukhari, "Rheumatoid arthritis: recent advances on its etiology, role of cytokines and pharmacotherapy," Biomedicine \& Pharmacotherapy, vol. 92, pp. 615-633, 2017.

[34] M. Sajid, M. R. Khan, S. A. Shah et al., "Investigations on antiinflammatory and analgesic activities of Alnus nitida Spach (Endl). stem bark in Sprague Dawley rats," Journal of Ethnopharmacology, vol. 198, pp. 407-416, 2017.

[35] K. Amagase, N. Izumi, Y. Takahira, T. Wada, and K. Takeuchi, "Importance of cyclooxygenase-1/prostacyclin in modulating gastric mucosal integrity under stress conditions," Journal of Gastroenterology and Hepatology, vol. 29, pp. 3-10, 2014.

[36] A. L. Cornett and C. S. Lutz, "Regulation of COX-2 expression by miR-146a in lung cancer cells," $R N A$, vol. 20, no. 9, pp. 1419-1430, 2014.

[37] H.-Y. Yoon, E.-G. Lee, H. Lee et al., "Kaempferol inhibits IL$1 \beta$-induced proliferation of rheumatoid arthritis synovial fibroblasts and the production of COX-2, PGE2 and MMPs," International Journal of Molecular Medicine, vol. 32, no. 4, pp. 971-977, 2013.

[38] L. J. Crofford, R. L. Wilder, A. P. Ristimäki et al., "Cyclooxygenase- 1 and -2 expression in rheumatoid synovial tissues. Effects of interleukin-1 beta, phorbol ester, and corticosteroids," Journal of Clinical Investigation, vol. 93, no. 3, pp. 1095-1101, 1994.

[39] A. Dickman and J. Ellershaw, "For Discussion NSAIDs: gastroprotection or selective COX-2 inhibitor?" Palliative Medicine, vol. 18, no. 4, pp. 275-286, 2004.
[40] P. Vitale, S. Tacconelli, M. G. Perrone et al., "Synthesis, pharmacological characterization, and docking analysis of a novel family of diarylisoxazoles as highly selective cyclooxygenase-1 (COX-1) inhibitors," Journal of Medicinal Chemistry, vol. 56, no. 11, pp. 4277-4299, 2013.

[41] T. J. P. Hla, "Molecular characterization of the $5.2 \mathrm{~KB}$ isoform of the human cyclooxygenase-1 transcript," Prostaglandins, vol. 51, no. 1, pp. 81-85, 1996.

[42] J. Roca-Ferrer, M. Pérez-Gonzalez, F. J. Garcia-Garcia et al., "Low prostaglandin E2and cyclooxygenase expression in nasal mucosa fibroblasts of aspirin-intolerant asthmatics," Respirology, vol. 18, no. 4, pp. 711-717, 2013. 